Tohoku J. exp. Med., 1981, 135, 345-347

\title{
Distribution of Sensory Nerve Endings in the Labial Mucosa of the Mouse
}

\author{
Tetsu Yamamoto and Sanya Sakada \\ Department of Physiology, Tokyo Dental College, Chiba 260
}

\begin{abstract}
Yamamoto, T. and Sakada, S. Distribution of Sensory Nerve Endings in the Labial Mucosa of the Mouse. Tohoku J. exp. Med., 1981, 135 (4), 345-347 The distribution of sensory nerve endings in the labial mucosa of the mouse was investigated by the vital methylene blue staining method. Encapsulated corpuscles were distributed uniformly throughout the mucosa, whereas bush-like nerve endings were localized in the area along the median line where the mucosal epithelium showed a considerable thickness. The number of the encapsulated corpuscles per individual was $72-133$, and that of the bush-like nerve endings was 131-248. sensory nerve endings; oral mucosa; lip; mouse
\end{abstract}

Various sensory nerve endings have been observed in the oral mucosa of the human and other mammals and their morphological characteristics have been described in detail (Winkelmann 1959; Malinovsky 1966; Seto 1972). Little information is available, however, on the distribution and density of the sensory nerve endings involved in the sensitivity of the oral mucosa. The present study was undertaken to examine quantitatively the distribution of the sensory nerve endings in the labial mucosa of the mouse.

\section{Methods}

After thirty-five albino mice weighing 28-36 $\mathrm{g}$ were anesthetized with ether, the hairy skin of the lower lips were removed thoroughly to expose the submucosa and the fresh dissected tissues including all the area of the labial mucosa were prepared for light microscopy. The specimens were stained with a $0.01 \%$ methylene blue solution for $15 \mathrm{~min}$ at $37^{\circ} \mathrm{C}$, fixed in chilled $8 \%$ ammonium molybdate solution for $12 \mathrm{hr}$ and washed by running water for $30 \mathrm{~min}$. Whereas five specimens were embedded in paraffin and prepared into $25 \mu \mathrm{m}$ serial sections cut vertically to the mucosal surface to investigate the position of the nerve endings in the mucosa, other specimens were mounted wholly on glass slides in glycerin to examine the distribution pattern, the number per individual and the density of the nerve endings.

\section{Results and Discussion}

In the labial mucosa of the mouse, there are two kinds of organized sensory nerve endings, encapsulated corpuscles and bush-like nerve endings, in addition to free nerve endings.

The encapsulated corpuscles (Fig. 1A), with an inner core and a capsule around the axon are almost oval in shape, 10-40 $\mu \mathrm{m}$ in diameter and $30-120 \mu \mathrm{m}$ in

Received for publication, February 19, 1981. 
length. According to the appearance of the axon, the encapsulated corpuscles vary from the simple type similar to the simple paciniform corpuscle (Malinovsky 1966) or mammalian end-organ (Winkelmann 1959) to the complex type resembling the mucocutaneous end-organ (Winkelmann 1959). The fiber diameter of these corpuscles at the distal internodal segment is $3.0-6.0 \mu \mathrm{m}$. The encapsulated corpuscles are located in the lamina propria at a distance from the epithelium, and distributed uniformly throughout the labial mucosa (Fig. 2A). The number of these corpuscles per individual was $72-133$ and the density in the entire mucosa was $8-16 / \mathrm{mm}^{2}$.

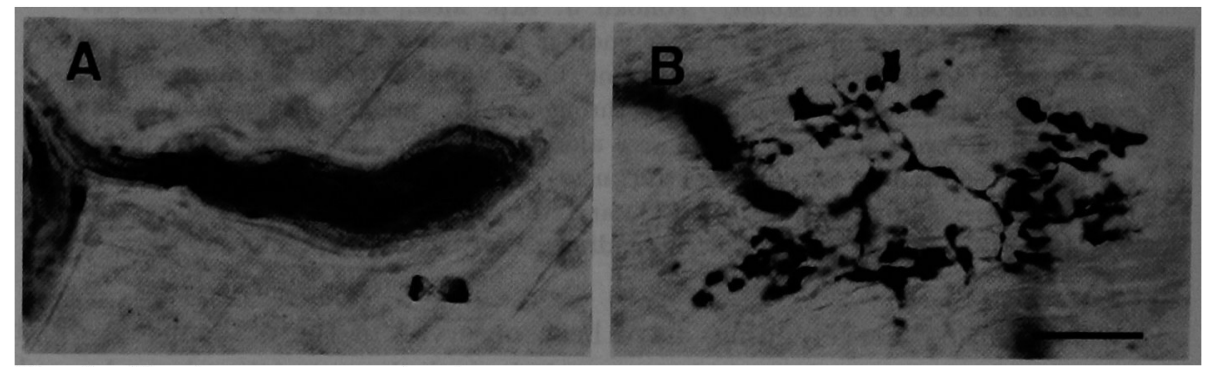

Fig. 1. Simple type encapsulated corpuscle (A) and a bush-like nerve ending (B) in the labial mucosa of the mouse. Vital methylene blue staining. Scale: $20 \mu \mathrm{m}$.

A

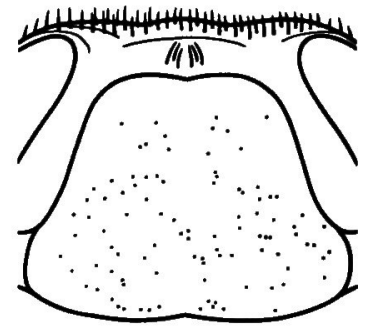

B

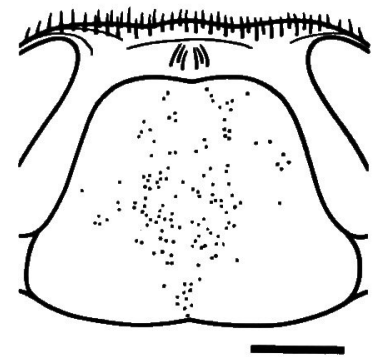

Fig. 2. Schematic diagrams showing the distributions of encapsulated corpuscles (A) and bush-like nerve endings (B). Scale: $1 \mathrm{~mm}$.

The bush-like nerve endings (Fig. 1B) have no capsule, and the axon shows a complex aspect of arborization. The ramification of the axon of these endings is more complex than that of branched terminations in the human oral mucosa (Seto 1972) and the fiber diameter is 3.0-4.5 $\mu \mathrm{m}$. The bush-like nerve endings are situated in the lamina propria close to the epithelium. Their axons, however, are not contacting the epithelium. These endings are localized within the long and narrow area of the mucosa along the median line where the epithelium is well developed and the formation of papillae is remarkable (Fig. 2B). It is considered, therefore, that there is a close relationship between the specialization of the bush-like nerve ending and the development of the epithelium. The number of the bushlike nerve endings per individual was 131-248 and the densities in the areas where these endings concentrated amounted to high values $66-118 / \mathrm{mm}^{2}$. 


\section{References}

1) Malinovský, L. (1966) The variability of encapsulated corpuscles in the upper lip and tongue of the domestic cat (Felis ocreata L., f. domestica). Folia morphol., 14, 175-191.

2) Seto, H. (1972) The sensory innervation of the oral cavity in the human fetus and juvenile mammals. In: Third Symposium on Oral Sensation and Perception, edited by J.F. Bosma, C.C. Thomas, Springfield, pp. 35-75.

3) Winkelmann, R.K. (1959) The erogenous zones: Their nerve supply and its significance. Proc. Mayo Clin., 34, 39-47. 